\title{
Low Electron Energy Loss Spectroscopy of Fluorescent Probes
}

\author{
R.L. Felts, ${ }^{*}$ V. Seybold, $* *$ \\ * Carl Zeiss NTS, LLC, One Corporation Way, Peabody, MA 01960. \\ ** Carl Zeiss NTS, GmbH, Oberkochen, Germany.
}

Absorption and excitation energies of many dyes, fluorophores and naturally colored biological substances that are routinely imaged in the Light Microscope are found in the low electron energy loss region (1.5-3.5eV) (Fig. 1). Due, however, to electron microscope limitations these have been underutilized as a method to image specific biological regions of interest. In 2007 Ottensmeyer and co-workers published their findings of the distribution of the anti-cancer drug doxorubicin (DOX) in human breast cancer cells MCF-7 imaged directly using low energy loss electron microscopy[1]. Their worked was carried out using a prism-mirror-prism imaging electron energy filter coupled to a previous generation Zeiss EM902. For the work presented here we used a Zeiss Libra 200 with a monochromated (MC) FEG ion source and in-column doubly corrected Omega type electron energy filter.

The analytical capabilities of the instrument in EELS are given by the combination of the stable doubly corrected in-column energy filter, and the FEG monochromator. At $200 \mathrm{kV}$ the energy resolution of the total system measured at the full width half maximum (FWHM) of the zero-loss peak has a specified value of $200 \mathrm{meV}$ but can be as small as $41 \mathrm{meV}$ for very short exposure times $(0.03 \mathrm{~s})$. This resolution displays only a slight increase to $50 \mathrm{meV}$ when the accelerating voltage is lowered to $80 \mathrm{kV}$ and the exposure time is extended to 1s. These energy loss resolutions allow for accurate measurement of the low-loss energy signals of fluorescent probes that were used in this research.

Fluorescent probes suspended in solution were applied to a carbon coated copper grids with a 600 mesh hole size The excess liquid was wicked away leaving a thin homogenous layer of material on the grid surface. An EELS spectrum was collected for each sample and the low-loss energy peak was identified. These peaks located in the $1.5-3.5 \mathrm{eV}$ range can be difficult to distinguish for the pi* peaks, $5-10 \mathrm{eV}$, but due to the small zero-loss energy resolution of the Libra $200 \mathrm{MC}$ it is easily recognizable.

References

[1] A. Mhawi et al. Journal of Structural Biology 158 (2007) 80-92. 

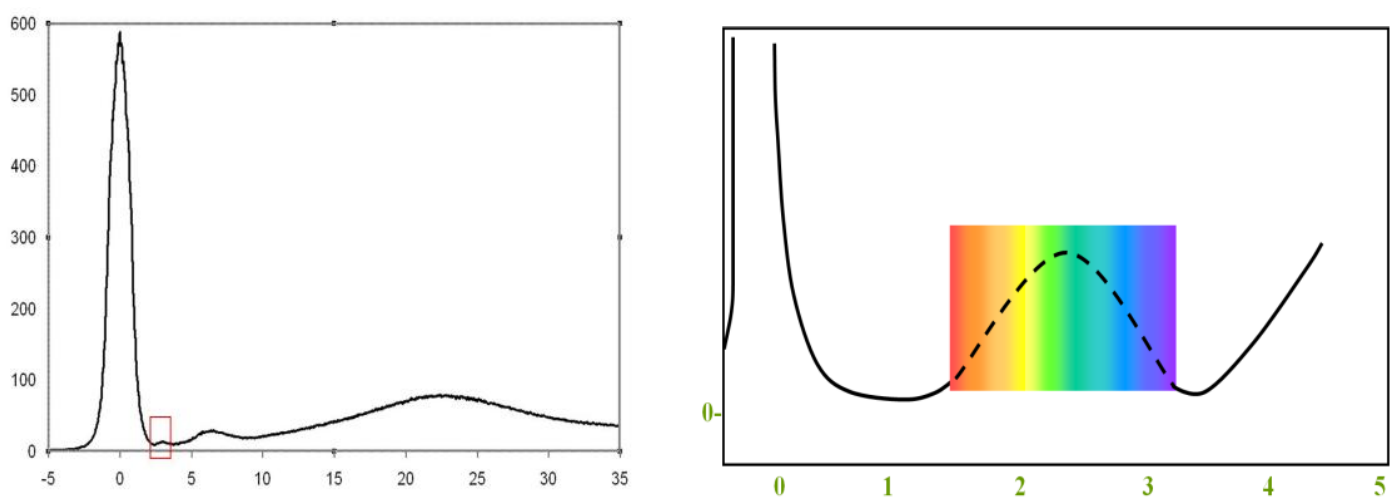

FIG 1. Typical Electron Energy Loss Spectrum. In (a) the zero loss peak, the low-loss fluorescence peak, the pi* transition peak and the sigma* plasmon are all visible. The red box is a zoomed region of the fluorescence peak highlighted in figure (b). In (b) a visible spectrum has been overlaid on the low-loss fluorescence peak. 